\title{
Burnishing of a non-axisymmetric curved surface with a CNC lathe
}

\author{
Keigo TAKASUGI*, Masayoshi SHINYA**, Yoshiya ISOZAKI***, Naohiko SUZUKI****, \\ Yoshiyuki KANEKO****, Naoki ASAKAWA* and Yoshitaka MORIMOTO***** \\ * Institute of Science and Engineering, Kanazawa University \\ Kakuma, Kanazawa, Ishikawa 920-1192, Japan \\ E-mail: ktaka@se.kanazawa-u.ac.jp \\ ** Industrial Research Institute of Ishikawa \\ 2-1, Kuratsuki, Kanazawa, Ishikawa 920-8203, Japan \\ *** YAMAHA MOTOR Co., Ltd. \\ 2500 Shingai, Iwata, Shizuoka 438-8501, Japan \\ **** Technical Division, Takamatsu Machinery Co., Ltd. \\ 1-8, Asahigaoka, Hakusan, Ishikawa 925-8558, Japan \\ ***** Department of Mechanical Engineering, Kanazawa Institute of Technology \\ 3-1 Yatsukaho, Hakusan, Ishikawa 924-0838, Japan
}

Received: 12 May 2021; Revised: 17 June 2021; Accepted: 29 June 2021

\begin{abstract}
The present study proposes a novel burnishing process called non-axisymmetric curved surface (NACS)-burnishing using a lathe that can machine a non-axisymmetric curved surface, such as a cam for internal combustion engines, with high efficiency. In the present paper, the offset in the Y-direction, which is a unique machining condition for NACS-burnishing, is first described. In this machining method, since the contact point of the burnishing tool changes continuously, the apparent rake angle of the burnishing tool also changes continuously, and its sign affects the finished surface properties. Therefore, it will be shown that the rake angle can be negative in the entire machining region by setting an offset of the tool in the Y-direction based on the geometrical relationship between the tool and the workpiece, and the conditional equation of the rake angle is derived. Next, actual NACS-burnishing experiments are carried out, and the effectiveness of the method is verified in terms of surface roughness and residual stress with the availability of the offset in the Y-direction. In particular, although tearing occurs when there is no offset, it is confirmed that the torn surface can be eliminated by the offset. Thereby, a surface roughness Ra of less than $0.2 \mu \mathrm{m}$ and a compressive residual stress of over $200 \mathrm{MPa}$ can be obtained for hardened steel with HRC60, which has sufficient surface properties.
\end{abstract}

Keywords: Burnishing, Non-axisymmetric surface, Turning, Diamond tool, CAM, Residual stress

\section{Introduction}

Recently, the machining accuracy, strength, and corrosion resistance of automotive mechanical parts, such as cams for internal combustion engines, must reach a high level, and machining must be performed at low cost. Since sliding under high pressure is essential for materials used in engine cams, etc., high wear resistance and satisfactory surface roughness are necessary. In general, in order to obtain high wear resistance, hardened steels with an HRC of over 50 to 55 are used, and finish machining with grinding targeting an Ra of 0.25 to $0.75 \mu \mathrm{m}$ is required (Karamış et al., 2012). In addition, it is difficult to machine engine cams, which are non-circular, i.e., have non-axisymmetric profiles. Therefore, manufacturing of engine cams depends on specialized grinding centers (Morimoto et al., 2014), and the increased costs associated with specialization and complicated compensation of the grinding wheel prevent the improvement of productivity. As such, Morimoto et al. (Morimoto et al., 2012; Takasugi et al., 2018) developed 

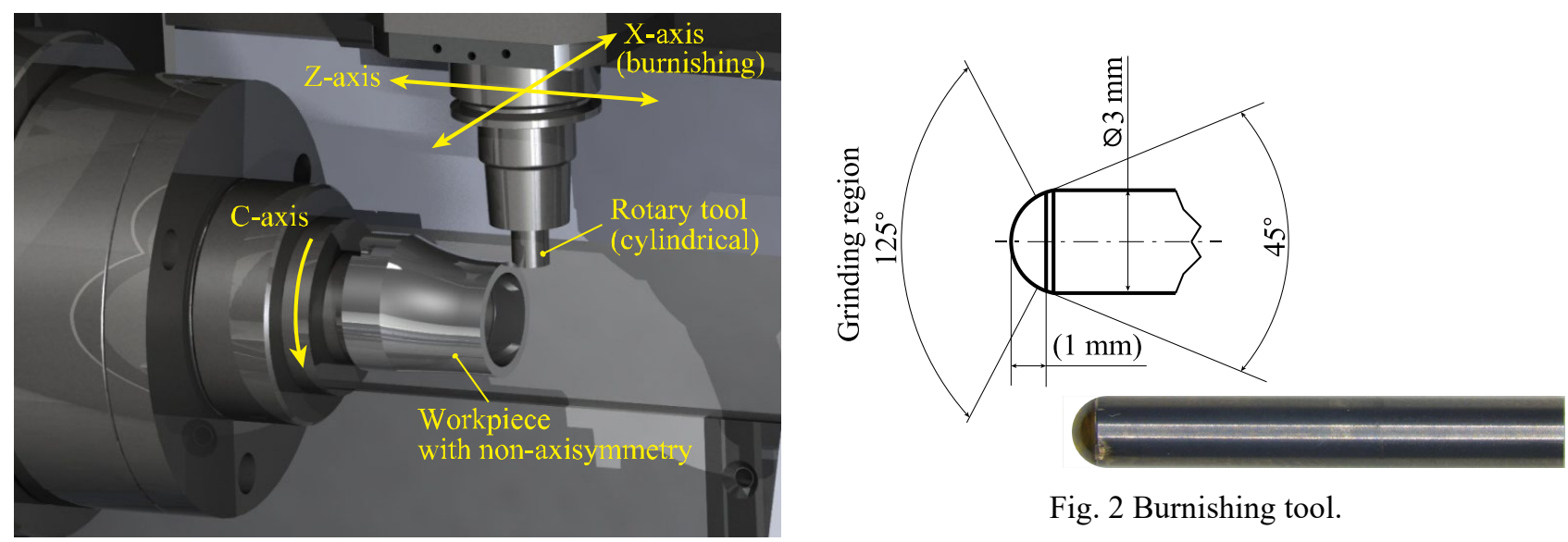

Fig. 2 Burnishing tool.

Fig. 1 Photograph of non-axisymmetric curved surface (NACS)-turning. When burnishing, the tool is attached along the X-direction.

non-axisymmetric curved surface (NACS)-turning as a new machining method. Although NACS-turning has the same axis configuration as the conventional $\mathrm{CNC}$ lathe, non-axisymmetric curved surface machining by turning is enabled by synchronizing the turning spindle and the $\mathrm{X}$-axis driven by a linear motor. Thus, significant improvement of productivity with respect to non-axisymmetric curved surface profiles is expected.

On the other hand, burnishing with high productivity is attracting attention as a method by which to obtain a high surface roughness (Loh and Tam, 1988). In burnishing, a satisfactory surface roughness is obtained by pressing and sliding a roller or ball shaped tool against the workpiece under constant pressure. In addition, improvements in the surface hardness, fatigue strength, and wear resistance are expected by the addition of work hardening and residual stress, which leads to an improvement in productivity. In particular, the diamond burnishing tool has been reported to be effective as a high-hardness-material tool, and is the subject of the present study. Tobala et al. (2015a, 2015b, 2017) and Tanaka et al. (2009) investigated the effectiveness of burnishing of tool steels and harden steels with an HRC exceeding 60 using diamond chips. Liska et al. (2012) verified the relationship among the feed rate, the burnishing force, and the surface roughness in the burnishing process with a diamond chip. In addition, several studies on the burnishing of free-form surfaces have recently been conducted. For example, Lee et al. (1992) performed ball burnishing of a free-form surface using a four-axis machining center and clarified that the surface roughness depends on the pressing depth, burnishing speed, and feed rate. Okada et al. (2017) developed a 5-DOF parallel mechanism that can control both the posture of the tool and the pressing force and carried out burnishing with a constant force. Based on these studies, the ability of burnishing was clarified and it is expected to be an acceptable replacement for conventional grinding in some cases.

Based on the above considerations, the present study proposes a new burnishing method using NACS-turning (NACS-burnishing), which can be applied to non-axisymmetric curved surface profiles at a high machining speed, similar to that of turning. In the present study, a burnishing tool specialized for NACS-burnishing is developed, and burnishing experiments are carried out for several machining conditions. As a result of verifying the properties of burnishing surface, the same surface roughness and residual stress as in conventional burnishing are confirmed while maintaining high machining productivity by the NACS-turning mechanism. In addition, offset of the burnishing tool in the tool axis direction affects surface properties as a unique parameter for NACS-burnishing.

\section{Non-axisymmetric curved surface-burnishing}

First, an outline of NACS-turning is described here. As shown in Fig. 1, NACS-turning has three translation axes: $\mathrm{X}, \mathrm{Y}$, and $\mathrm{Z}$. The $\mathrm{X}$-axis indicates the cutting depth, and the Z-axis indicates the feed direction. Finally, the Y-axis indicates the direction orthogonal to the $\mathrm{X}$ - and $\mathrm{Z}$-axes. Moreover, the $\mathrm{C}$-axis is the rotation axis of the workpiece. This setup is the same as that for conventional CNC lathes. As mentioned in Section 1, the X-axis is driven by the linear motor synchronizing the rotation of the $\mathrm{C}$-axis. Then, the $\mathrm{X}$-axis can be controlled in a period of $0.222 \mathrm{~ms}$. For example, when the $\mathrm{C}$-axis rotates at $375 \mathrm{~min}^{-1}$, positioning orders of 720 times in one rotation can be executed by $\mathrm{CNC}$. 
Takasugi, Shinya, Isozaki, Suzuki, Kaneko, Asakawa and Morimoto, Journal of Advanced Mechanical Design, Systems, and Manufacturing, Vol.15, No.5 (2021)

Table 1 Specifications of non-axisymmetric curved surface-turning.

\begin{tabular}{c|c}
\hline \hline Maximum spindle speed $\left[\mathrm{min}^{-1}\right]$ & 2,000 \\
\hline Thrust force in the X-direction $[\mathrm{N}]$ & 3,600 \\
\hline Maximum acceleration in the X-direction $\left[\mathrm{m} / \mathrm{s}^{2}\right]$ & 114 \\
\hline Control period $[\mathrm{ms}]$ & 0.222 \\
\hline
\end{tabular}

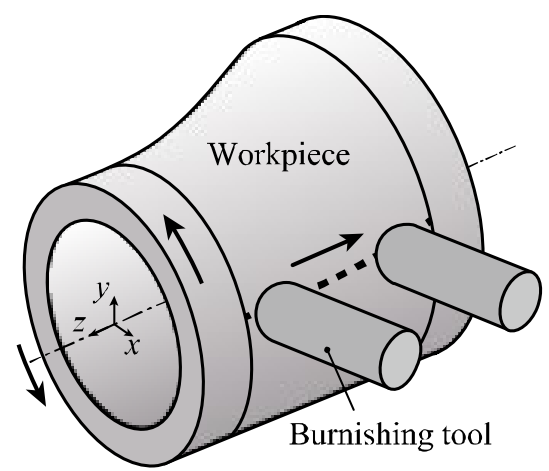

Fig. 3 Relative position between the burnishing tool and the workpiece.

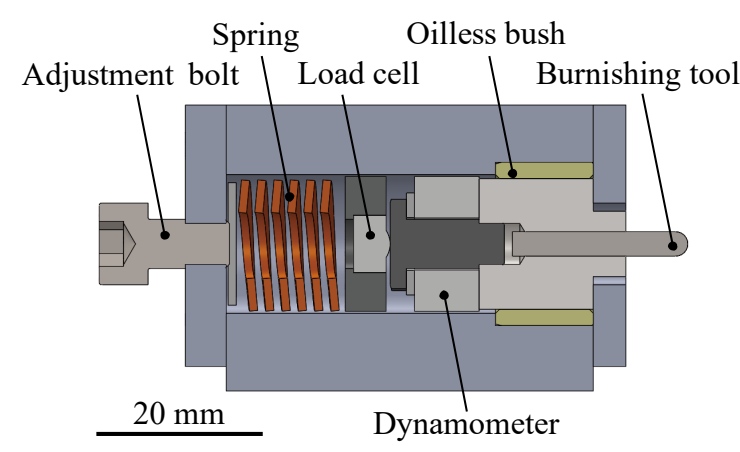

Fig. 4 Components of the burnishing tool.

Therefore, the position of the tool tip can be determined at an interval of $0.5^{\circ}$, and non-axisymmetric profiles can be machined. A cylindrical cutting tool that has a cutting edge was installed for NACS-turning.

In burnishing using a lathe for NACS-turning, a burnishing tool having a spherical head is installed instead of a cylindrical cutting tool. Figure 2 shows the burnishing tool installed in the present study. A partial spherical single-crystal diamond is brazed to cemented carbide having a diameter of $3 \mathrm{~mm}$. Then, since the grinded region of the diamond is only $125^{\circ}$, it is impossible to set the tool in the Z-direction considering the contact area between the tool and the workpiece. Therefore, the tool-axis direction is set as corresponding to the $\mathrm{X}$-axis, as shown in Fig. 3. Moreover, the burnishing tool is constructed of components, as shown in Fig. 4. Since burnishing under constant pressure is a general technique, the burnishing tool in the present study is supported by a spring pre-loaded by a built-in load cell, and the burnishing tool slides along the workpiece profile by means of the spring reaction force. Furthermore, the component of the burnishing tool is designed to avoid resonance between the spring-mass system and the workpiece rotation speed. A dynamometer is also implemented in the burnishing tool, and it is possible to measure the dynamic pressing force of the tool during burnishing.

Finally, the primary specifications of NACS burnishing are listed in Table 1.

\section{Machining characteristics}

In NACS-burnishing, not only the general conditions of burnishing, such as pressing force and feed rate, but also the following two conditions derived from the peculiarity of NACS-burnishing have to be considered in order to obtain a good machined surface property.

(i) Moving direction of $\mathrm{X}$-axis

(ii) Tool offset in the Y-direction

The $\mathrm{X}$-axis moves conforming with the non-axisymmetric workpiece profile at high speed. Then, when the distance from the rotation center of the workpiece to the contact point between the tool and the workpiece increases, the X-axis moves in the direction of tool pulling. Instead, when the distance decreases, the X-axis moves in the direction of tool pushing, as shown in Fig. 5. These two situations lead to different burnishing conditions. Figure 6 shows the pulling and pushing situations modeled by the two-dimensional cutting theory. In the pulling situation, since the tool falls forward in the rotation direction, the rake angle $\alpha$ becomes negative. Then, thrust force $F_{t}$, which is one component force of the friction force, $\boldsymbol{F}$, applied to the tool, is applied in the direction pushing the tool (Fig. 6(a)). Conversely, in the pushing situation, $\alpha$ becomes positive and $\boldsymbol{F}_{t}$ is applied in the direction of pulling the tool (Fig. 6(b)). Since the tool is supported by the spring force, the instantaneous decrease in the amount of the tool suppressing force becomes large for the case in which the burnishing tool passes over a large bump, such as a turning mark, when pushing, which can 


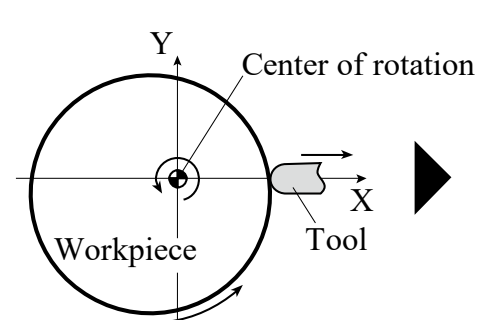

(a) Pulling

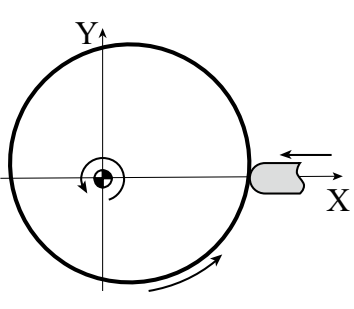

(b) Pushing

Fig. 5 Moving direction along the X-axis according to the workpiece profile.
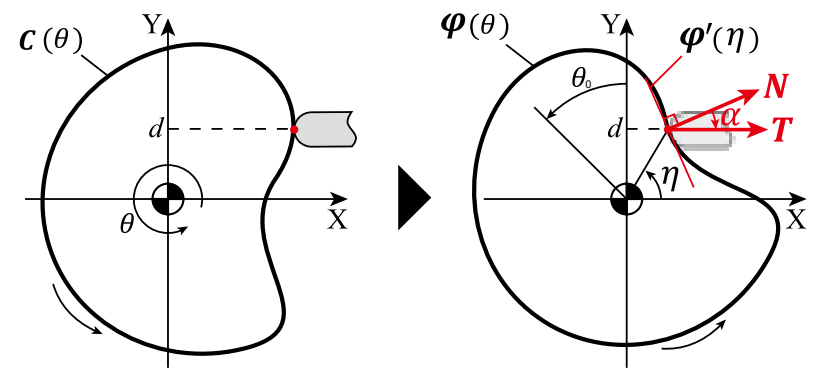

Fig. 7 Definition of parameters in NACS-burnishing to obtain the relationship between offset $d$ and rake angle $\alpha$.

lead to deterioration of the burnished surface property. This situation is referred to herein as the unstable burnishing process.

In order to avoid unstable burnishing in the pushing situation, it is effective to set a tool offset in the Y-direction. The proof is described below. First, the workpiece profile is set as $\boldsymbol{C}(\theta)$ in a fixed X-Y coordinate system. Then, $\theta$ is the rotation angle around the rotation axis. When the profile in which $C$ rotates by $\theta_{0}$ is set as $\boldsymbol{\varphi}(\theta), \boldsymbol{\varphi}$ is described as follows:

$$
\boldsymbol{\varphi}(\theta)=\left[\begin{array}{cc}
\cos \theta_{0} & -\sin \theta_{0} \\
\sin \theta_{0} & \cos \theta_{0}
\end{array}\right] \boldsymbol{C}(\theta)
$$

As shown in Fig. 7, when an offset in the Y-direction, $d$, is set, and the angle, $\eta$, between the contact point and the X-axis is defined, the tangential vector on the contact point is represented as $\varphi^{\prime}(\eta)$. Therefore, the normal vector $N$ on the contact point is defined as rotated by $90^{\circ}$ :

$$
\boldsymbol{N}=\left[\begin{array}{cc}
0 & 1 \\
-1 & 0
\end{array}\right] \boldsymbol{\varphi}^{\prime}(\eta)
$$

In addition, $\boldsymbol{T}=\left(\begin{array}{ll}1 & 0\end{array}\right)^{t}$ because the direction of the tool is the same as the X-axis. Moreover, $\alpha$ is defined as the angle between $\boldsymbol{N}$ and $\boldsymbol{T}$, and $-2 / \pi<\alpha<2 / \pi$ considering the condition of the actual rake angle. Accordingly, it is better to confirm the positivity or negativity of $\alpha$ using $\sin \alpha$. Then, we have

$$
\sin \alpha=\boldsymbol{N} \times \boldsymbol{T}=C^{\prime}(\eta)_{y} \sin \theta_{0}-C^{\prime}(\eta)_{x} \cos \theta_{0}, \quad\left(\left|\boldsymbol{C}^{\prime}(\eta)\right|=1\right)
$$

where subscripts $x$ and $y$ indicate the components of a vector. Then, as shown in Fig. 7, when $\boldsymbol{T}$ becomes greater than $\boldsymbol{N}$ in the clockwise direction, $\alpha>0$ and $\boldsymbol{N} \times \boldsymbol{T}>\mathbf{0}$. Moreover, $\alpha$ becomes negative, i.e., stable burnishing occurs.

Moreover, the relationship between $d$ and $\eta$ is represented as follows:

$$
\boldsymbol{\varphi}(\eta)_{y}=d
$$

Expanding Eq. (4) yields 
Takasugi, Shinya, Isozaki, Suzuki, Kaneko, Asakawa and Morimoto, Journal of Advanced Mechanical Design, Systems, and Manufacturing, Vol.15, No.5 (2021)

Table 2 Experimental conditions.

\begin{tabular}{c|l}
\hline \hline \multirow{2}{*}{ Workpiece } & $\begin{array}{l}\text { SKD11 (hardened, HRC60), } \\
\varnothing 35 \mathrm{~mm}(\text { eccentricity: } 3 \mathrm{~mm})\end{array}$ \\
\hline \multirow{2}{*}{ Tool } & $\begin{array}{l}\text { Brazed diamond tip } \\
\text { (SCD, Ra: } 0.008 \mu \mathrm{m})\end{array}$ \\
\hline
\end{tabular}

\begin{tabular}{c|l}
\hline Spindle speed $\left[\mathrm{min}^{-1}\right]$ & 375 \\
\hline Feed rate $[\mathrm{mm} / \mathrm{rev}]$ & 0.05 \\
\hline Pressing force $[\mathrm{N}]$ & $40,60,100,150,200$ \\
\hline Burnishing length $[\mathrm{mm}]$ & 5 \\
\hline Offset $[\mathrm{mm}]$ & 0,5 \\
\hline
\end{tabular}

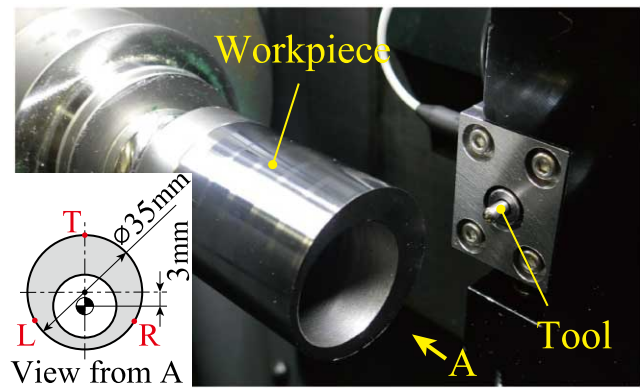

Fig. 8 Experimental setup.

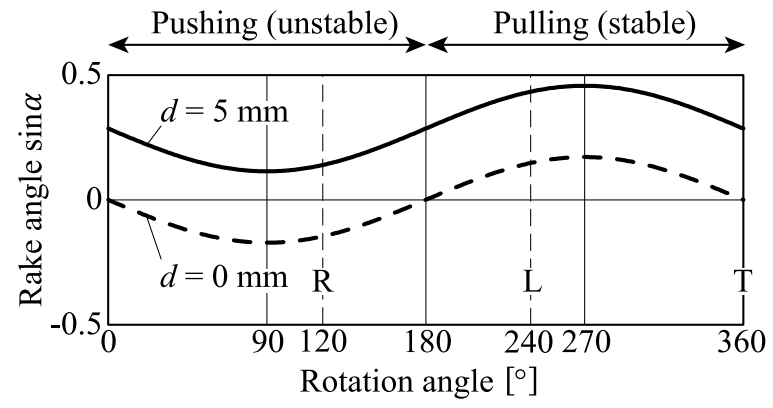

Fig. 9 Comparison of change in rake angle during a rotation with and without offset.

$$
\boldsymbol{C}(\eta)_{x} \sin \theta_{0}+\boldsymbol{C}(\eta)_{y} \cos \theta_{0}=d
$$

Substituting Eq. (5) into Eq. (3) yields

$$
\sin \alpha=\left(C_{y}^{\prime}+\frac{C_{x}}{C_{y}} C_{x}^{\prime}\right) \sin \theta_{0}-\frac{C_{x}^{\prime}}{C_{y}} d
$$

In order to satisfy $\alpha>0$, the solution of Eq. (6) must be greater than 0 . Finally,

$$
d>\frac{c_{y}^{\prime}}{c_{x}^{\prime}} C_{y}+C_{x} .
$$

Therefore, $\alpha$ becomes negative in one rotation by adopting $d$ satisfying Eq. (7), and stable burnishing can be performed.

\section{Experiment}

\subsection{Condition}

Experiments were carried out to verify the effectiveness of NACS-burnishing. The experimental conditions are shown in Table 2, and an overview of the actual experimental setup is shown in Fig. 8. As mentioned in Section 2, the spindle speed is fixed at $375 \mathrm{~min}^{-1}$ in order to synchronize the rotation and X-axis movement for a pitch of $0.5^{\circ}$, and a burnishing tool with a diameter of $3 \mathrm{~mm}$ in which a single-crystal diamond is brazed to the tool tip is used. The feed rate is also fixed at $0.05 \mathrm{~mm} / \mathrm{rev}$, and the burnishing length in the Z-direction is $5 \mathrm{~mm}$. The workpiece material is SKD 11 , which is assumed as the material for an engine cam. At this time, the workpiece profile is a circle of $35 \mathrm{~mm}$ in diameter considering the ease of the verification. However, axisymmetry is realized by shifting the center of rotation 3 $\mathrm{mm}$ from the center of the circle. In order to verify the difference in surface quality by offset $d$, comparison of the cases with/without $d$ in the + Y-direction was carried out. The eccentric circle profile is expressed as 


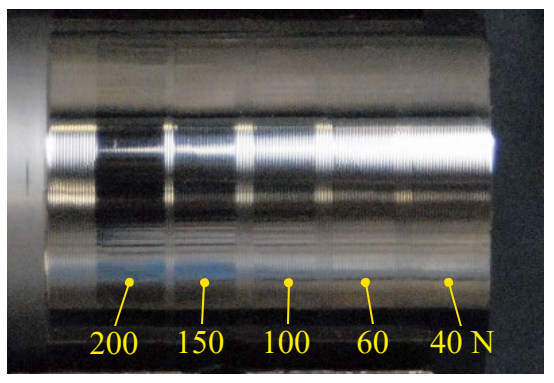

Fig. 10 Appearance of the burnished workpiece for various pressing forces $(d=0 \mathrm{~mm})$.

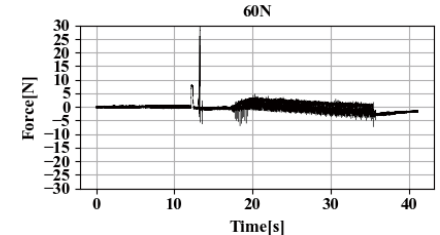

$150 \mathrm{~N}$

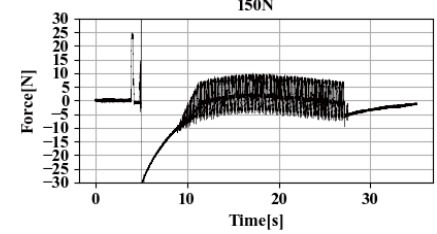

Fig. 11 Measurement results of thrust force by dynamometer during burnishing for various pressing forces $(d=0 \mathrm{~mm})$.

$$
\boldsymbol{C}(\theta)=\left(\begin{array}{c}
r \cos \theta+\varepsilon \\
r \sin \theta
\end{array}\right)
$$

where $r$ is the radius, and $\varepsilon$ is the eccentricity. From Eqs. (7) and (8), $d>\varepsilon$ is derived. Since $\varepsilon=3 \mathrm{~mm}$ in the workpiece profile of the experiment, $d>3 \mathrm{~mm}$. Therefore, $d=5 \mathrm{~mm}$ is adopted in this experiment. The theoretical change in $\alpha$ during one rotation when $d=0$ or 5 is shown in Fig. 9.

Since the burnished surface property depends on the pressing force of the tool, five pressing forces 40, 60, 100, 150 , and $200 \mathrm{~N}$ are examined in this experiment. These pressing forces are preset using the built-in load cell under the condition that the workpiece is stationary. The experimental results are verified based on microscopic image, surface roughness $\mathrm{Ra}$ and $\mathrm{Rz}$, and residual stress. Surface roughness and residual stress are measured in the axial and circumferential directions, respectively. Residual stress is measured using the $\cos \alpha$ method (Sasaki et al., 2013).

As an advantage, burnishing can add compressive residual stress to the workpiece surface. For example, shot peening is in the same category of minute plastic deformation processing and can add a compressive residual stress of approximately 200 to $300 \mathrm{MPa}$ (Akiniwa et al., 2003). It is difficult to set a target value of compressive residual stress without reservation in the burnishing process because various conditions affect the results. However, it is better to give higher compressive residual stress in general. Therefore, in the present study, the target compressive residual stress in NACS-burnishing is also set as the same value for shot peening. The residual stress was measured at three points, T, L, and $\mathrm{R}$, and divided into three equal angles in increments of $120^{\circ}$, as shown in the Fig. 8. Moreover, the measurement directions at each point were axial and circumferential directions. Pulling and pushing situations occur at points $\mathrm{L}$ and $\mathrm{R}$, respectively. Point $\mathrm{T}$ is an inflection point that indicates switching from pulling to pushing.

\subsection{Result and discussion $(d=0 \mathrm{~mm})$}

The experimental results for the case in which $d=0 \mathrm{~mm}$ are shown in Figs. 10 to 14 . Figure 10 shows the appearance of the burnished workpiece surface. The surface with only turning was prepared for comparison with the burnishing surfaces. Figure 11 shows the AC components of forces in the tool axis direction (thrust) during burnishing by the built-in dynamometer. Although static waves form, i.e., an amplitude of 0 is ideal, amplitudes of approximately $10 \%$ from preset static pressing forces were actually observed in every graph. This is assumed to be caused by the synchronization error between the order value of $\mathrm{CNC}$ and actual movement. However, it is assumed that force variations of approximately $10 \%$ are within the allowance of NACS-burnishing, because the burnished surface had no effect, as shown in Figs. 12 to 14.

Microscopic images of burnished surfaces for each thrust force and each measurement part are shown in Fig. 12. In the figure, when the pressing force is $60 \mathrm{~N}$ and the measurement point is L, the caption is described as " $60 \mathrm{~N} \_$L." The results for $40 \mathrm{~N}$ are omitted from the figure for the sake of clarity. It is confirmed that the turning marks shown in the images of the criteria turning surfaces gradually become weaker as the thrust force increases and vanish at $150 \mathrm{~N}$ to 200 N. However, torn surfaces at point R, as shown at 60N_R, 100N_R, and 150N_R, are observed. Actually, torn surfaces can be also confirmed at 40N_R and 200 N_R slightly through the feel of the surfaces. Therefore, it is assumed that tears occur at positive rake angles and deteriorate according to the increase of the pressing force. However, this phenomenon diminishes when the pressing force exceeds a certain value. Moreover, tearing occurs intensively at 


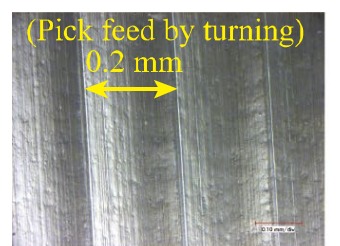

Turning_ $\mathrm{T}$

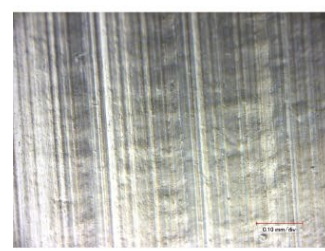

Turning_L

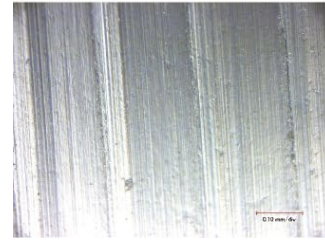

Turning_R

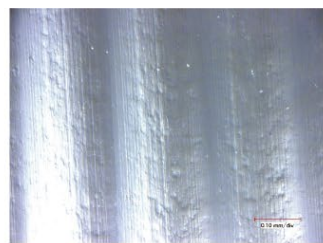

$60 \mathrm{~N} \_\mathrm{T}$

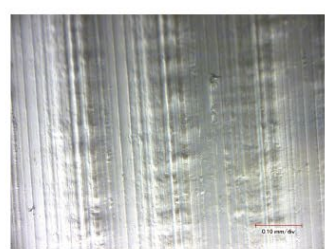

60N_L

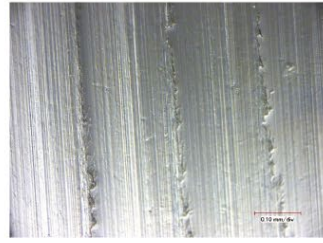

$60 \mathrm{~N} \mathrm{R}$

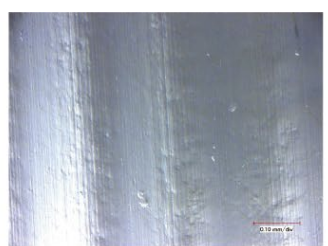

$100 \mathrm{~N} \mathrm{~T}$

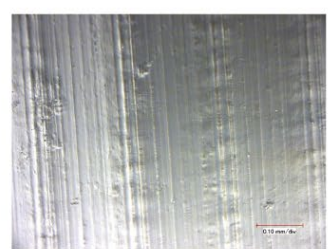

100N_L

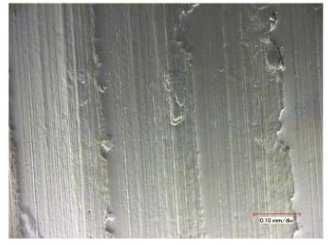

$100 \mathrm{~N} \mathrm{R}$

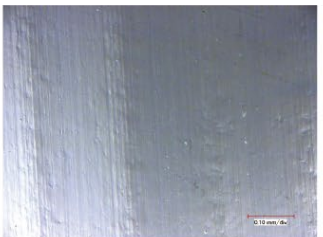

$150 \mathrm{~N} \mathrm{~T}$

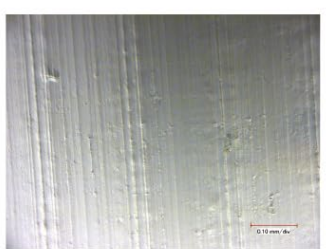

150N_L

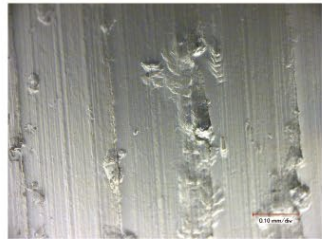

$150 \mathrm{~N} \mathrm{R}$

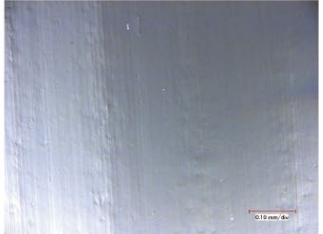

200N_T

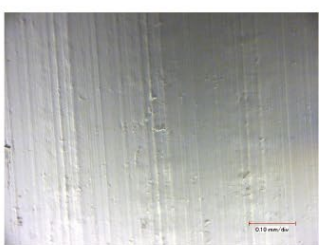

200N_L

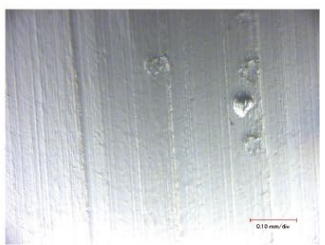

$200 \mathrm{~N} \mathrm{R}$

Fig. 12 Microscopic image of the turned and burnished surface for each thrust force and measurement $(d=0)$.
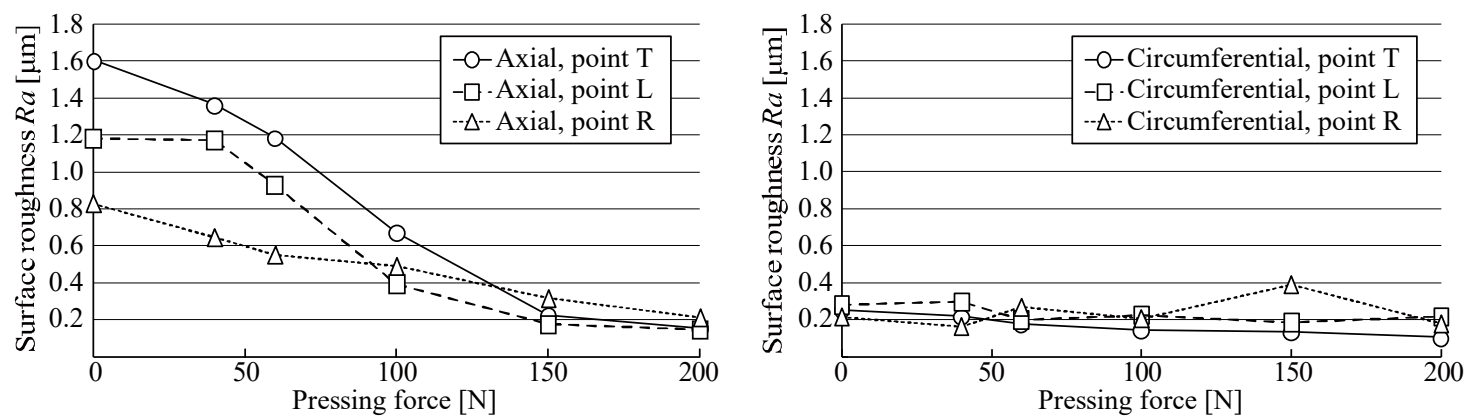

Fig. 13 Surface roughness for each thrust force and measurement $(d=0 \mathrm{~mm})$.
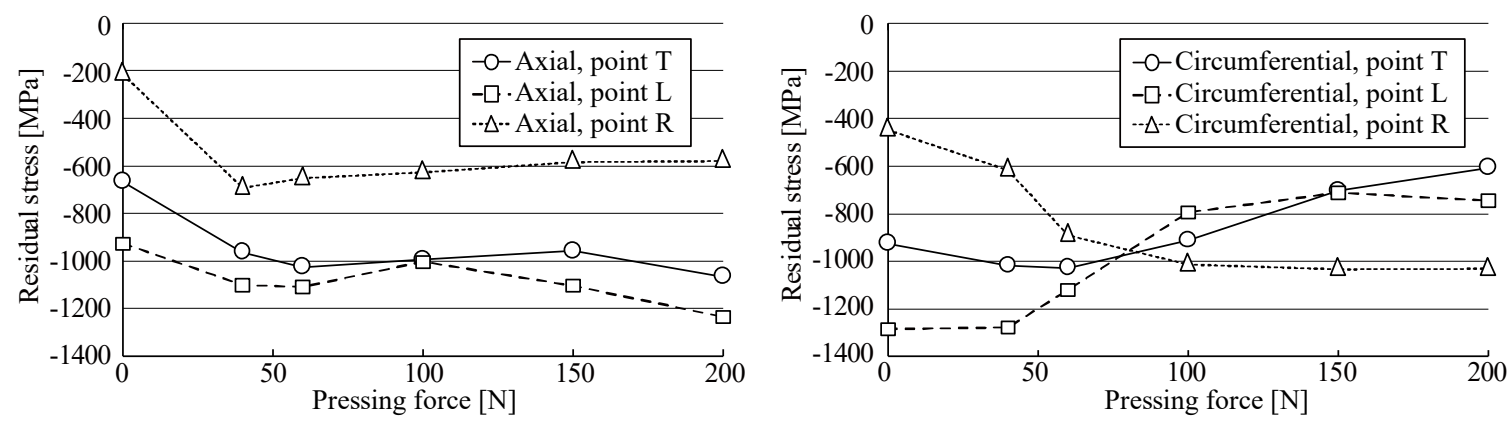

Fig. 14 Residual stress for each thrust force and measurement $(d=0 \mathrm{~mm})$.

convex edges between turning marks. Based on the above, it is considered that unstable vibration, such as the stick-slip phenomenon, occurs at the R part where $\boldsymbol{F}_{t}$ is applied in the strip-off direction of the tool, and tearing on the surfaces occurs when the tool passes the convex edge of the turning mark, as mentioned in Section 3.

The measurement results for surface roughness are shown in Fig. 13. A pressing force of $0 \mathrm{~N}$ indicates the criteria turning surfaces. Improvement of the surface roughness due to an increase in the pressing force is confirmed for all measurement location and both measurement directions. In particular, approximately $\mathrm{Ra}=0.2 \mu \mathrm{m}$, corresponding to a grinding finish surface, was achieved under the condition of $200 \mathrm{~N}$. However, slight deterioration of the surface roughness due to the effect of tearing is also confirmed at $150 \mathrm{~N}$ for the circumferential conditions and at point $\mathrm{R}$.

The measurement results for the residual stress are shown in Fig. 14. Negative values indicate compressive residual stress. In NACS-turning, as in the setup shown in Fig. 1, the cutting tool initially has a negative rake angle. Therefore, it is assumed that the criteria turning surface already has compressive residual stress to some extent. In the axial 


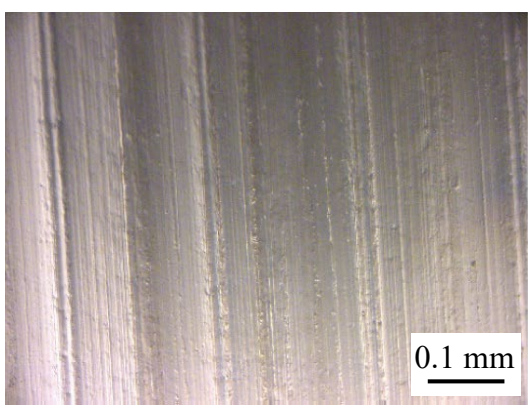

(a) $100 \mathrm{~N}$

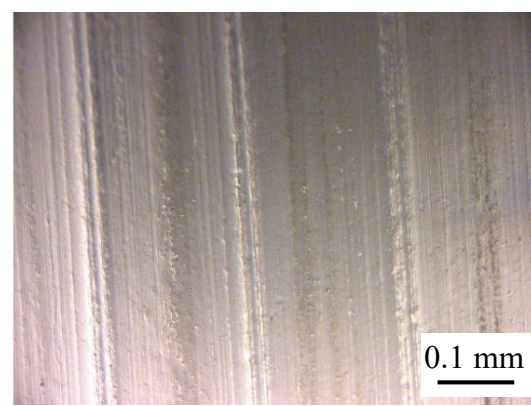

(b) $150 \mathrm{~N}$

Fig. 15 Microscopic images of the burnished surface (point $\mathrm{R}, d=5 \mathrm{~mm}$ ).

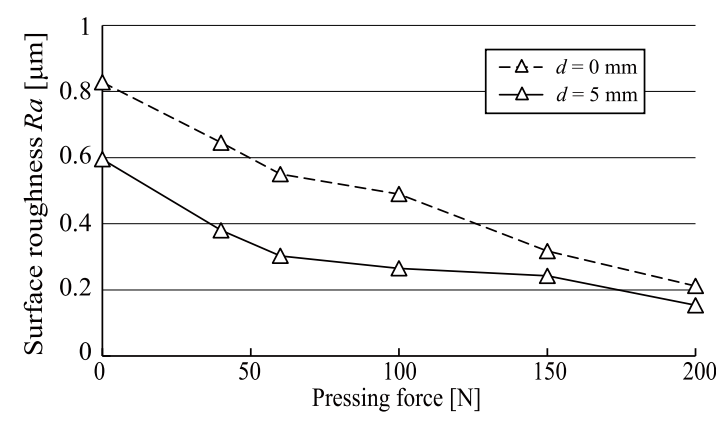

(a) Axial

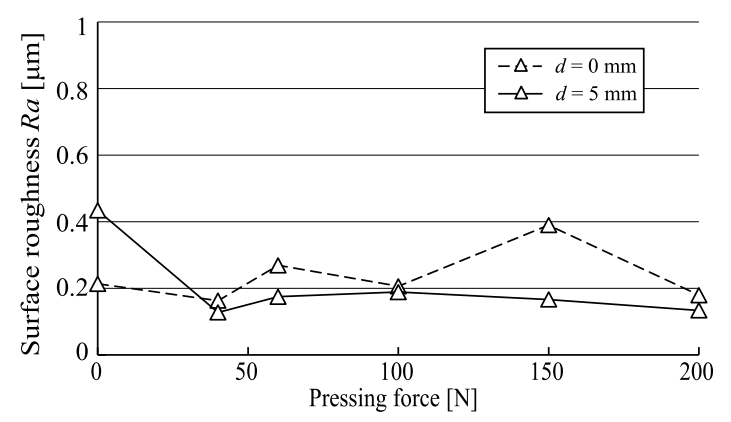

(b) Circumferential

Fig. 16 Surface roughness for $d=0$ and $5 \mathrm{~mm}$ at point $\mathrm{R}$.

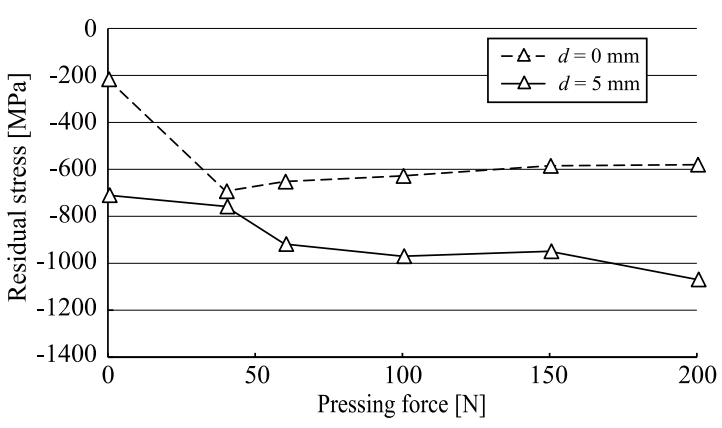

(a) Axial

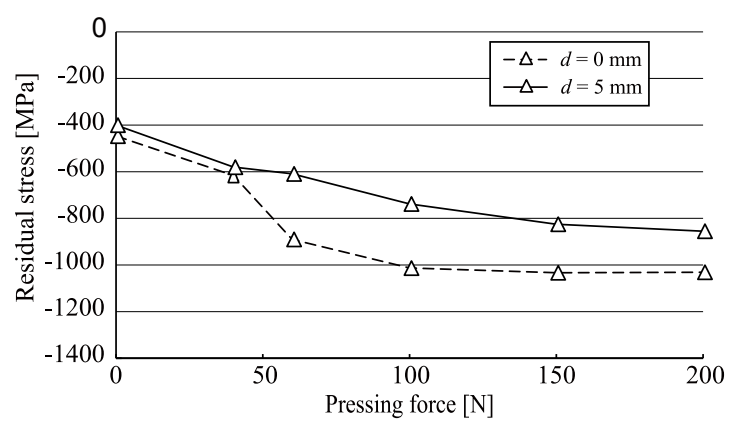

(b) Circumferential

Fig. 17 Residual stress for $d=0$ and $5 \mathrm{~mm}$ at point $\mathrm{R}$.

direction, an increase in compressive residual stress is confirmed. Moreover, a large compressive residual stress, e.g., over $600 \mathrm{MPa}$, that cannot be obtained by general turning or grinding. However, in the circumferential direction, the compressive residual stress tends to become weaker at points $\mathrm{T}$ and $\mathrm{L}$. It is assumed that this tendency is related to the direction of metal flow during burnishing. In the burnishing process, plastic deformed metal by tool feeding flows around the tool. Therefore, convex shapes called scallops are formed by metal flow beside the tool, i.e., in the axial direction instead of the circumferential direction, and the scallops are burnished in the next rotation. Thus, irregular stress may have occurred on the scallops, and unexpected variations in the residual stress were observed. Since X-ray can penetrate only slightly beyond the surface of the workpiece, the residual stress in the depth direction is uncertain. However, a sufficient compressive stress could be obtained even after burnishing. Verification of the actual fatigue strength, for example, is intended as an area for future research.

In summary, the surface roughness can be reduced under a high pressing force of over $150 \mathrm{~N}$, except for a few areas in which the rake angle becomes negative. Furthermore, the expected compressive residual stress can be maintained throughout the entire burnished region, although the surface obtained by NACS-turning already has a high compressive residual stress.

In the next section, the measurement results for the case in which the offset $(d=5 \mathrm{~mm})$ is prepared as a countermeasure against the torn surface are described. 


\subsection{Result and discussion $(d=5 \mathrm{~mm})$}

The same experiments and measurements were carried out for $d=5 \mathrm{~mm}$ in order to verify the effect of the rake angle on the finish surface. Figures 15 through 17 show the measurement results for point $\mathrm{R}$, where considerable tearing is observed. As shown in Fig. 15, it is confirmed that tearing shown in Fig. 12 at 60N_R to 150N_R is eliminated as expected. An improvement can be also found in the measurement results of surface roughness, as shown in Fig. 16. The surface roughness is improved slightly in both directions. In particular, although the surface roughness exhibited dispersion in the results for $d=0 \mathrm{~mm}$ by tearing (Fig. 16(b)), the dispersion is suppressed for $d=5 \mathrm{~mm}$. Moreover, compressive residual stress over the target value is confirmed for the measurement results in Fig. 17. Therefore, it is found that there is an association between tearing and the amount of residual stress. However, typically, residual stress occurs because a material is constrained. Namely, if a crack or a tear occurs, then the stress is released and tends to be zero. Therefore, it is difficult to conclude how the mechanism of tearing is applied at this moment, and this will be examined in a future study.

Based on the above results, it is clarified that NACS-burnishing can maintain a negative rake angle by adopting an offset in an advantageous direction.

\section{Conclusion}

In the present paper, NACS-burnishing, which is a novel burnishing method using a lathe for NACS-turning, is proposed. The following results are obtained by developing and verifying the effectiveness of NACS-burnishing:

(i) A tool for NACS-burnishing that can provide a constant pressing force by a spring was generated, and it is confirmed that burnishing while maintaining a constant pressing force and a $10 \%$ variation in terms of the rotation speed in $375 \mathrm{~min}^{-1}$ is feasible.

(ii) In NACS-burnishing, it was clarified that the rake angle changes continuously depending on the geometrical relation at the contact point between the tool and the workpiece profile, and it is shown that a negative rake angle can be maintained throughout the entire region by applying the offset in the Y-direction.

(iii) As a result of the experiment in NACS-burnishing, a surface roughness Ra of $0.2 \mu \mathrm{m}$ was achieved for a pressing force of over $150 \mathrm{~N}$. Moreover, a sufficient compressive residual stress was confirmed.

(iv) Tearing found in some conditions can be improved by adopting the offset in the Y-direction.

\section{References}

Akiniwa, Y., Tanaka, K., Suzuki, K., Yanase, E., Nishio, K., Kusumi, Y., Okado, H. and Arai, K., Evaluation of residual stress distribution in shot-peened steel by synchrotron radiation, Journal of the Society of Materials Science, Japan (in Japanese), Vol.52, No.7 (2003), pp.764-769.

Karamış, M. B., Cerit, A. A., Selçuk, B. and Nair, F., The effects of different ceramics size and volume fraction on wear behavior of Al matrix composites (for automobile cam material), Wear, Vol.289, June (2012), pp.73-81.

Lee, S. S. G., Tam, S. C. and Loh, N. H., An investigation into the ball burnishing of an AISI 1045 free-form surface, Journal of Materials Processing Technology, Vol.29, No.1-3 (1992), pp.203-211.

Líska, K., Kodácsy, J. and Líska, J., Investigation of the microgeometry after hard turning and diamond burnishing, Advanced Materials Research, Vol.472-475, February (2012), pp.902-907.

Loh, N. H. and Tam, S. C., Effects of ball burnishing parameters on surface finish -A literature survey and discussion-, Precision Engineering, Vol.10, No.4 (1988), pp.215-220.

Morimoto, Y., Nakagaki, K., Moriyama, T., Saito, H. and Takasugi, K., Development of high-acceleration moving table for machining tools (Evaluation of high speed motion of tandem table arranged on same axis), Transactions of the JSME (in Japanese), Vol.80, No.818 (2014), pp. 1-14.

Morimoto, Y., Emoto, S., Moriyama, T., Kato, H., Nakagaki, K., Suzuki, N., Kaneko, Y. and Isobe M., Creation of curved surface by lathe turning -Development of CAM system using original tool layout-, Procedia CIRP, Vol. No.1 (2012), pp.114-119.

Okada, M., Shinya, M., Matsubara, H., Kozuka, H., Tachiya, H., Asakawa, N. and Otsu, M., Development and characterization of diamond tip burnishing with a rotary tool, Journal of Materials Processing Technology, Vol.244, June (2017), pp.106-115. 
Sasaki, T., Maruyama, Y., Ohba, H. and Ejiri, S., Two-dimensional imaging of Debye-Scherrer ring for tri-axial stress analysis of industrial materials, $15^{\text {th }}$ International Workshop on Radiation Imaging Detectors, June (2013), pp.1-9.

Takasugi, K., Morimoto, Y., Kaneko, Y., Suzuki, N. and Asakawa, N., Improvement of machining accuracy for 3D surface machining with CNC lathe, Journal of Advanced Mechanical Design, Systems, and Manufacturing, Vol.12, No.4 (2018), pp.1-14.

Tanaka, H., Tabuto, H., Yanagi, K. and Futamura, M., Effect of surface hardened steel texture of preliminary process on burnishing process - a metrological study of hardened steel surface finishing using diamond burnishing tool, Journal of the Japan Society for Technology of Plasticity, Vol.50, No.581 (2009), pp.555-559.

Tobala, D., Brostow, W., Czechowski, K. and Rusek, P., Improvement of wear resistance of some cold working tool steels, Wear, Vol.382-383, No.15 (2017), pp.29-39.

Tobala, D., Brostow, W., Czechowski, K., Rusek, P. and Wronska, I., Structure and properties of burnished and nitride AISI D2 tool steel, MTERIALS SCIENCE, Vol.21, No.4 (2015a), pp.511-516.

Tobola, D., Rusek, P., Czechowski, K., Miller, T. and Duda, K., New indicators of burnished surface evaluation reasons of application, Metrology and Measurement Systems, Vol.22, No.2 (2015b), pp.263-274. 